\title{
Interruption and Reversal of Direct Oral Anticoagulants in Preprocedural and Acute Settings
}

\author{
Abhishek Kulkarni, MD, FAAC and Megha Manek, MD
}

With increasing physician and patient awareness, the use of direct oral anticoagulants (DOACs) has been steadily increasing in recent years. Unfortunately, given their recent introduction, the reversal of anticoagulation attained with DOACs is not well studied. Given the diverse mechanisms of action of these newer agents, no universal antidote is available for reversal. Laboratory assays that can accurately quantify the anticoagulation status of patients on DOAC therapy are not readily available, which presents a challenge in situations demanding rapid reversal. Furthermore, the safety and efficacy of reversal agents have not been extensively investigated in the clinical setting.

This review briefly summarizes commercially available DOACs, delineates current knowledge related to reversal of DOAC agents in specific clinical settings, and identifies areas of future study. ( $\mathrm{J}$ Am Board Fam Med 2018;31:817-827.)

Keywords: Anticoagulants, Blood Coagulation

New direct oral anticoagulants (DOACs) have emerged as a popular alternative to traditional vitamin $\mathrm{K}$ antagonists (VKAs) across several clinical settings. New DOACs accounted for $62 \%$ of all new prescriptions in the United States and $98 \%$ of all anticoagulant-related costs between 2010 and 2013. ${ }^{1}$ The commercially available DOACS in the United States are apixaban, dabigatran, rivaroxaban, betrixaban, and edoxaban. As DOAC-based anticoagulation becomes more prevalent, so does the need for appropriate reversal strategies and antidotes.

\section{Indications for DOAC Therapy}

DOAC-based anticoagulation is used across multiple clinical scenarios, which necessitate the use of oral anticoagulation. The 2 most common scenar-

This article was externally peer reviewed.

Submitted 7 January 2018; revised 30 April 2018; accepted 14 May 2018.

From Division of Cardiology, Southern Illinois University School of Medicine, Springfield, IL (AK); Community \& Family Medicine, Southern Illinois University School of Medicine, Springfield (MM).

Funding: none.

Conflict of interest: none declared.

Corresponding author: Abhishek Kulkarni, MD, FAAC, Division of Cardiology, Southern Illinois University School of Medicine, 751 N. Rutledge Street, Springfield, IL 62702 (E-mail: akulkarni52@SIUMED.edu). ios for DOAC therapy are in the management of venous thromboembolism (VTE) and in the prevention of stroke and systemic emboli in patients with nonvalvular atrial fibrillation (NVAF).

\section{DOACs in NVAF}

Atrial fibrillation is associated with a 5-fold increase in the risk of stroke and is the most common preventable cause of stroke. ${ }^{2-4}$ Patients with valvular atrial fibrillation (VAF) should be anticoagulated with VKA. Patients with NVAF should undergo risk stratification based on the $\mathrm{CHA}_{2} \mathrm{DS}_{2}$ VASc score. Patients with a score of $\geq 2$ are considered to be at moderate to high risk for stroke and would benefit from anticoagulation. ${ }^{5}$ Five major NVAF trials have compared the efficacy and safety of DOACS to VKA (target international normalized ratio [INR], 2 to 3): $\mathrm{RELY}^{6}$ for dabigatran, ROCKET-AF ${ }^{7}$ for rivaroxaban, $\mathrm{AVERROES}^{8}$ and ARISTOTLE $^{9,10}$ for apixaban, and ENGAGE$\mathrm{AF}^{11}$ for edoxaban. These phase 3 trials resulted in the approval of these agents for commercial use for NVAF. The European consensus documents ${ }^{12,13}$ on choosing a particular anticoagulant and dosage for stroke prevention in NVAF discusses the choice of DOAC and dosing for special treatment groups. These recommendations are summarized in Table 1. ${ }^{14}$ 
Table 1. Anticoagulation for Atrial Fibrillation in Specific Clinical Scenarios ${ }^{14}$

\begin{tabular}{|c|c|}
\hline Category & Recommendations \\
\hline Stable CAD and PAOD & -DOAC mostly without use of antiplatelet agents \\
\hline \multirow[t]{2}{*}{ After coronary stenting } & $\begin{array}{l}\text {-Triple therapy with VKA (target INR, } 2 \text { to } 2.5 \text { ) or reduceddose DOAC*, aspirin } 75 \text { to } \\
100 \mathrm{mg} / \mathrm{d} \text {, and clopidogrel } 75 \mathrm{mg} / \mathrm{d}\end{array}$ \\
\hline & $\begin{array}{l}\text {-Consider: bare metal stents, abbreviated 6-month dual antiplatelet therapy for drug } \\
\text { eluting stent or omission of aspirin if bleeding propensity is high }\end{array}$ \\
\hline \multirow[t]{2}{*}{ Secondary stroke prevention } & -DOACs preferred over VKA unless TTR $>70$ \\
\hline & -No addition of antiplatelet agent to OAC is needed. \\
\hline \multirow[t]{2}{*}{ Acute stroke } & $-\mathrm{r}-\mathrm{tPA}$ only if anticoagulation by test or history is minimal \\
\hline & -Mechanical thrombectomy for proximal intracranial occlusion \\
\hline \multirow{4}{*}{$\begin{array}{l}\text { Acute ischemic stroke after } \\
\text { neuroimaging (repeat imaging pre- } \\
\text { OAC for moderate to severe } \\
\text { ischemic stroke) }\end{array}$} & - TIA start OAC immediately \\
\hline & - Mild ischemic stroke, start OAC after 3 days \\
\hline & - Moderate ischemic stroke, start OAC at 5 to 7 days \\
\hline & - Severe ischemic stroke, start OAC at 12 to 14 days \\
\hline History of GI bleed & -Preference to apixaban and low dose dabigatran \\
\hline Hemodialysis & - VKA or no OAC (DOACs not approved if $\mathrm{CrCl}<15 \mathrm{~mL} / \mathrm{m}$ ) \\
\hline Cardioversion & -VKA and DOACS appear to be similarly effective \\
\hline AF- Ablation & -Preferred VKA over DOACS (limited data on edoxaban) \\
\hline Mechanical valves & $\begin{array}{l}\text {-VKA target INR based on valve type, site and associated conditions along with aspirin } \\
75 \text { to } 100 \mathrm{mg} \text { daily }\end{array}$ \\
\hline Mod/severe rheumatic mitral stenosis & VKA target INR 2 to 3 \\
\hline
\end{tabular}

CAD, coronary artery disease; DAOC, direct oral anticoagulants; GI, gastrointestinal; AF, atrial fibrillation; TTR, Time in Therapeutic Range; OAC, oral anticoagulants; TIA; transient ischemic attack; r-tPA, recombinant tissue plasminogen activator; PAOD, peripheral arterial occlusive disease; VKA, vitamin $\mathrm{K}$ antagonists.

* Apixaban $2.5 \mathrm{mg}$ BID.

\section{DOACs in VTE}

VTE is a diagnosis that typically consists of deep vein thrombosis (DVT) and/or acute pulmonary embolism (PE). Anticoagulation is the cornerstone of the treatment for patients with VTE. The fundamental principle of anticoagulation is the prevention of further thrombosis and complications. Previously, the only oral anticoagulant group approved for this role was VKAs. However, multiple recent trials have demonstrated the noninferiority of DOACs to VKA, prompting their widespread use. EINSTEIN-DVT ${ }^{15}$ and EINSTEIN-PE ${ }^{16}$ were open-label randomized trials that demonstrated the noninferiority of rivaroxaban to conventional therapy in the management of VTE. AMPLIFY ${ }^{17}$ was a prospective, randomized, double-blind trial that compared apixaban with conventional anticoagulation for the treatment of acute VTE (DVT and/or $\mathrm{PE})$. There was no difference in the rates of recurrent symptomatic VTE or VTE-related death between the groups. Edoxaban, an oral factor $\mathrm{Xa}$ inhibitor, one of the newest DOACs to be approved by the Food and Drug Administration (FDA), was proven to be noninferior to the conventional treatment of acute VTE in a randomized, double-blind study. ${ }^{18}$ RECOVER I was a prospective, randomized, double-blind trial that compared dabigatran (oral direct thrombin inhibitor) with the conventional treatment (VKA) for the management of acute VTE. Compared with VKAs, dabigatran had a similar incidence of recurrent VTE and VTE-related deaths. ${ }^{19}$

\section{DOACs in VTE Prevention}

The prevention of VTE events in multiple populations is an area of ongoing study and recommendations vary greatly based on the setting. Methods of VTE prevention include a spectrum of modalities, ranging from mechanical devices to heparin to DOAC therapy. The setting of treatment and the patient's risk for VTE also influence the choice of modality. ${ }^{20-22}$ In the postoperative period following orthopedic surgery, multiple clinical trials have demonstrated the efficacy of rivaroxaban, dabigatran, and apixaban in the prevention of VTE, which has led to their inclusion in the current CHEST guidelines for VTE prevention. ${ }^{21}$ In medically ill hospitalized patients, betrixaban is the only DOAC that is FDA approved for VTE prevention. ${ }^{23}$ 


\section{Safety of DOAC Therapy}

Therapeutic anticoagulation in any form has been associated with multiple complications, most common of which are major or minor bleeding complications. As with VKA in the past, DOAC therapy has been proven to increase rates of bleeding complications. Several predictive tools have been developed to identify risk of bleeding complications in patients on therapeutic anticoagulation. These tools include HEMORR2HAGES, HAS-BLED, RIETE, and ATRIA. The HAS-BLED score incorporates hypertension, abnormal renal/liver function, stroke, bleeding history, labile INR, elderly, and drug/alcohol use. A score of $\geq 3$ is suggestive of a high risk of bleeding complications. $^{24-26}$ The use of the HAS-BLED score is currently suggested by multiple guideline documents ${ }^{5,27}$ but has yet to be formally endorsed as part of clinical decision making regarding therapeutic anticoagulation. Notably, the HAS-BLED score has been validated in the atrial fibrillation population alone.

In patients with VTE, all the aforementioned DOAC trials included bleeding as a safety outcome. All the commercially available DOAC agents were associated with a numerically lower rate of major bleeding complications when compared with low molecular weight heparins and VKA. This reduction in bleeding events was moderate (in the case of dabigatran, rivaroxaban, and edoxaban) to robust (in the case of apixaban). ${ }^{27}$

In patients with NVAF, when compared with VKA, all DOACs reduced the risk of intracerebral, life-threatening, and fatal bleeds. Certain DOAC regimens demonstrated superiority in safety outcomes in these trials (dabigatran and apixaban). Furthermore, gastrointestinal bleeding may be higher with dabigatran, rivaroxaban, and edoxaban than with VKA therapy, although this has not been seen in patients with VTE. ${ }^{28-31}$ According to indirect comparisons, the risk of bleeding may be lower with apixaban than with the other DOACs. ${ }^{32}$

The clinical scenarios in which the utility of DOACs remain unclear are in the setting of prosthetic cardiac valves, VAF, and end-stage renal disease. In these settings, particularly that of end-stage renal disease, practice guidelines recommend using VKA preferentially. ${ }^{33}$ Limited evidence suggests that under exceptional circumstances that preclude the use of VKA in patients with end-stage renal disease on hemodialysis, apixaban can be used at a dose of $5 \mathrm{mg}$ twice daily (with a reduction in dose to $2.5 \mathrm{mg}$ twice daily for either age $\geq 80$ years or body weight $\leq 60 \mathrm{~kg}){ }^{34}$

\section{Interrupting and Reversing DOAV Therapy}

The most common scenarios that require aggressive reversal of DOAC therapy are major bleeding events or a need for emergent surgical procedures. In the past, the absence of specific antidotes or reversal agents made DOACs a less attractive option for patients and physicians. In the recent past, multiple agents that reverse the effects of DOAC therapy in a relatively short interval have been under development. However, the reversal of DOAC therapy/anticoagulation in an emergent setting requires a multifaceted approach. The type of response is often driven by the severity of the bleeding event or acuity of need for reversal.

\section{Nonemergent and Elective Invasive Procedures:}

Approximately $20 \%$ of patients on chronic anticoagulation require invasive or surgical procedures annually. ${ }^{35}$ It is important to understand that factors independent of the anticoagulation status of the patient can also influence bleeding risks. Significant among these factors are the inherent bleeding risk associated with the procedure ${ }^{36-38}$ and patient factors that can increase the risk for bleeding complications. $^{39-41}$

Procedures that are associated with a low risk of bleeding (such as minor dental, ophthalmologic, or dermatologic procedures) may be conducted without interrupting anticoagulation. ${ }^{42}$ In the subgroup of patients that require reversal of anticoagulation before a nonemergent surgical/invasive procedure, DOACs offer a theoretical advantage over VKA. Given the relatively short half-life of DOACs (6 to 17 hours), which is specific to each agent, the period of interrupting therapy before proceeding to surgery is generally shorter than VKA. However, the pharmacokinetics and elimination of DOACs are significantly influenced by renal function. Therefore, an assessment of renal function before deciding on the duration of interruption of DOAC therapy is essential. Unfortunately, given the paucity of robust trial data to support formal guidelines, clinical practice is often based on expert opinion and observational data. A recently published expert consensus document from the American 
College of Cardiology has laid out clinical pathways in this scenario. ${ }^{43}$ Given the absence of readily available laboratory testing to verify adequacy of reversal, this decision making must be individualized to each patient. Furthermore, given the relatively short duration of action of DOACs, there does not seem to be any role for using selective/ nonselective reversal agents.

\section{Preprocedural Management}

\section{A. Direct Thrombin Inbibitors}

Dabigatran, which is the only oral direct thrombin inhibitor, has a half-life of about 12 to 14 hours in patients with preserved renal function. In patients with renal dysfunction, the clearance of this medication is impaired, prolonging its half-life. ${ }^{44}$ Therefore, the duration of interruption of dabigatran depends on the patient's baseline renal function and the type of procedure. A specific protocol for the interruption of dabigatran before nonemergent surgery was studied and found to be promising. In this protocol, dabigatran was held preoperatively for a period that was determined based on the patient's creatinine clearance and the type of surgery planned. Based on these characteristics, dabigatran was held 24,48 , or 96 hours before the procedure. Of the 541 patients studied in the trial, only 1 patient experienced a thromboembolic complication in the form of a transient ischemic attack. ${ }^{45}$ Therefore, in patients with preserved renal function, undergoing procedures that are not associated with a high risk of bleeding, holding dabigatran 2 to 4 days before surgery seems to be a reasonable choice. In patients with abnormal renal function and those who are undergoing procedures associated with a higher bleeding risk and those requiring spinal/epidural anesthesia, dabigatran should be interrupted for a longer duration. Although patients receiving dabigatran have an elevated active partial thromboplastin time (aPTT), its utility as a surrogate for drug activity/anticoagulation has not been established. Instead, a diluted plasma thrombin time (if available) may be used to determine drug activity. ${ }^{46}$

Recommended durations for interrupting oral direct thrombin inhibitors, based on procedural bleeding risk and renal function (which alters the drug half-life), are summarized below in Table 2A.

\section{B. Factor Xa Inbibitors}

Rivaroxaban, apixaban, and edoxaban are the 3 commercially available factor $\mathrm{Xa}$ inhibitors. Although they belong to the same class, the pharmacokinetic properties of these agents are quite distinct, necessitating an individualized approach. Unfortunately, unlike direct thrombin inhibitors, there is little to no objective data to guide the management of factor $\mathrm{Xa}$ inhibitors. Hence, current practice relies of expert opinion and known pharmacokinetic characteristics of these agents. ${ }^{47}$

No routine coagulation analysis is helpful in determining adequacy of reversal of anticoagulation with these agents. In certain circumstances where the concern for bleeding is very high, antifactor Xa levels may help guide therapy. However, given the variability in the assays used by individual laboratories, their accuracy in the setting of oral factor Xa inhibitors should be confirmed before incorporating the results in clinical decision making.

Table 2A. Recommended Durations for Interrupting Oral Direct Thrombin Inhibitor in the Preprocedural Setting: Dabigatran $^{43,57,73}$

\begin{tabular}{lclc}
\hline & & \multicolumn{2}{c}{ Duration of Interruption } \\
\cline { 3 - 4 } $\begin{array}{c}\text { Creatinine Clearance } \\
\text { (ml/min) }\end{array}$ & $\begin{array}{c}\text { Estimated Half-Life } \\
\text { (hours) }\end{array}$ & Low PBR & $\begin{array}{c}\text { Intermediate/High/Uncertain } \\
\text { PBR }\end{array}$ \\
\hline & 13 & $\geq 24$ hours & $\geq 48$ hours \\
580 & 15 & $\geq 36$ hours & $\geq 72$ hours \\
30 to 49 & 18 & $\geq 48$ hours & $\geq 96$ \\
15 to 29 & 27 & $\geq 72$ hours & $\geq 120$ hours \\
$<15$ & 30 (off dialysis) & No data* Consider $\geq 96$ hours & No data \\
\hline
\end{tabular}

PBR, procedural bleeding risk.

${ }^{*}$ Consider measuring dilute thrombin time (dTT). 
a. Apixaban. The half-life of apixaban in patients with preserved renal function (creatinine clearance $>50 \mathrm{~mL} / \mathrm{min}$ ) receiving apixaban $5 \mathrm{mg}$ twice daily and patients with abnormal renal function (creatinine clearance $<50 \mathrm{~mL} / \mathrm{min}$ ) receiving apixaban $2.5 \mathrm{mg}$ twice daily is estimated at 8 to 15 hours. Therefore, apixaban may be held 2 to 3 days before a procedure (not associated with high bleeding risk). Longer durations of discontinuation may be warranted in patients undergoing procedures associated with high risk for bleeding or requiring spinal/epidural anesthesia.

b. Rivaroxaban. The half-life of rivaroxaban in patients with preserved renal function (creatinine clearance $>50 \mathrm{~mL} / \mathrm{min}$ ) receiving rivaroxaban 20 $\mathrm{mg}$ daily and patients with abnormal renal function (creatinine clearance of 30 to $50 \mathrm{~mL} / \mathrm{min}$ ) receiving rivaroxaban $15 \mathrm{mg}$ daily is estimated at 6 to 9 hours. Hence, rivaroxaban may be held 2 to 3 days before a procedure (not associated with high bleeding risk). Longer durations of discontinuation may be warranted in patients undergoing procedures associated with high risk for bleeding or requiring spinal/ epidural anesthesia.

c. Edoxaban. The half-life of edoxaban in patients with preserved renal function (creatinine clearance $>50 \mathrm{~mL} / \mathrm{min}$ ) receiving edoxaban $60 \mathrm{mg}$ daily and patients with abnormal renal function (creatinine clearance of 30 to $50 \mathrm{~mL} / \mathrm{min}$ ) receiving edoxaban $30 \mathrm{mg}$ daily is estimated at 6 to 11 hours. Hence, edoxaban may be held 2 to 3 days before a procedure (not associated with high bleeding risk). Longer durations of discontinuation may be warranted in patients undergoing procedures associated with high risk for bleeding or requiring spinal/epidural anesthesia.

Recommended durations for interrupting oral factor Xa inhibitors, based on procedural bleeding risk and renal function (which alters the drug half-life) are summarized below in Table 2B.

\section{Neuraxial Procedures:}

Bleeding complications, even of the smallest extent, in the setting of neuraxial procedures or neuraxial anesthesia can result in catastrophic complications. All DOACs carry a black box warning about their use in the setting of neuraxial anesthesia. Practice guidelines issued by the American Society of Regional Anesthesia and Pain Management advise longer interruptions preceding neuraxial procedures. The guidelines recommend an interruption of dabigatran 4 to 5 days before the procedure and an interruption of factor $\mathrm{Xa}$ inhibitors for 3 to 5 days. ${ }^{48}$ In light of the potential complications, the period of interruption may be prolonged further in the presence of extenuating circumstances, such as renal dysfunction and patients with high bleeding risks.

\section{Bridging Anticoagulation}

The use of bridging anticoagulation during and following periods of interruption of oral anticoagulation remains controversial and poorly studied, especially in DOACs. The limited data available (from study of VKA based anticoagulation) suggest that the risk of bleeding complications associated with bridging anticoagulation are significant. ${ }^{49}$ Given the relatively rapid onset and offset of anti-

Table 2B. Recommended Durations for Interrupting Oral Factor Xa Inhibitor in the Preprocedural Setting: Apixaban, Edoxaban, and Rivaroxaban ${ }^{57,74-76}$

\begin{tabular}{|c|c|c|c|}
\hline \multirow[b]{2}{*}{$\begin{array}{l}\text { Creatinine Clearance } \\
(\mathrm{ml} / \mathrm{min})\end{array}$} & \multirow[b]{2}{*}{$\begin{array}{c}\text { Estimated Half-Life } \\
\text { (hours) }\end{array}$} & \multicolumn{2}{|c|}{ Duration of Interruption } \\
\hline & & Low PBR & $\begin{array}{c}\text { Intermediate/High/Uncertain } \\
\text { PBR }\end{array}$ \\
\hline$\geq 30$ & 6 to 15 & $\geq 24$ hours & $\geq 48$ hours \\
\hline 15 to 29 & $\begin{array}{l}\text { Apixaban: } 17 \\
\text { Edoxaban: } 17 \\
\text { Rivaroxaban: } 9\end{array}$ & $\geq 36$ hours & $\begin{array}{l}\text { No data } \\
\text { Consider } \geq 72 \text { hours* }\end{array}$ \\
\hline$<15$ & $\begin{array}{l}\text { Apixaban: } 17^{\dagger} \\
\text { Edoxaban: } 10 \text { to } 17^{\dagger} \\
\text { Rivaroxaban: } 13^{\dagger}\end{array}$ & $\begin{array}{l}\text { No data } \\
\text { Consider } \geq 48 \text { hours }^{*}\end{array}$ & $\begin{array}{l}\text { No data } \\
\text { Consider } \geq 72 \text { hours* }\end{array}$ \\
\hline
\end{tabular}

PBR, procedural bleeding risk.

*Consider measuring agent-specific anti-Xa level.

${ }^{\dagger}$ Off dialysis. 
coagulation with DOAC agents compared with VKA, the duration of bridging is anticipated to be shorter. Despite the elevated risk of bleeding, there are specific circumstances under which bridging anticoagulation may be necessary. For instance, patients with a high risk of VTE in the setting of a recent venous thromboembolic event or a high CHADS $_{2}$-VASc score. ${ }^{50}$ Therefore, an appropriate risk-benefit analysis supplemented by an informed discussion with the patient may be prudent before pursuing bridging anticoagulation.

\section{Bleeding Complications and Urgent/Emergent Invasive Procedures}

In the setting of acute bleeding complications or urgent/emergent need for pursuing invasive procedures, the mere interruption of DOAC therapy is likely to be inadequate in avoiding blood loss and its associated complications. Although DOAC agents are short acting, restoration of normal coagulation parameters requires several hours after cessation of these agents. Despite the preponderance of evidence that supports the principle that DOACs cause less severe bleeding than VKAs, many patients and physicians were circumspect about initiating DOAC therapy in the absence of a specific reversal agent, particularly in the setting of a major bleeding event. ${ }^{51}$ With the recent advent of DOAC specific reversal agents, this perception is likely to change. At the outset, these catastrophic events require initial stabilization and supportive care as per typical protocols.

\section{Initial Management}

The preliminary management of major bleeding events or need for urgent/emergent surgery must include steps to stabilize the patient. In major bleeding events, advanced cardiac life support measures, addressing the site of blood loss, aggressive volume resuscitation, and transfusion of red blood cells and other blood products must be initiated immediately and should be continued concurrently with reversal of anticoagulation. ${ }^{36}$ If the DOAC agent has been ingested 2 to 4 hours preceding presentation, oral activated charcoal may be used to delay and disrupt the absorption of the agent. ${ }^{52}$ Another esoteric approach to eliminate the drug involves hemodialysis. Unfortunately, this strategy is effective only in patients who are on dabigatran. $^{53}$
As mentioned previously, the use of conventional coagulation parameters is of little to no value to guide management, especially in the acute setting. However, certain parameters are helpful for specific agents. A prolonged aPTT level may indicate that on-therapy level or clinically relevant level (that may contribute to bleeding) is present in patients taking dabigatran. Conversely, a thrombin time or a normal aPTT usually excludes clinically relevant levels of dabigatran. ${ }^{54} \mathrm{~A}$ prolonged PT suggests that on therapy or above on-therapy levels of apixaban, rivaroxaban or edoxaban are present. However, a normal PT does not rule out on-therapy or clinically relevant levels (especially if a relatively insensitive PT reagent is used). ${ }^{54}$

More specialized tests such as antifactor $\mathrm{Xa}$ levels, dilute thrombin time, ecarin chromogenic assay, and ecarin clotting time may be helpful but are less likely to be available in an emergent situation.

\section{Reversal Strategies}

The management of major bleeding complications or the reversal of anticoagulation in anticipation of an urgent or emergent invasive procedure can be broadly divided into nonspecific reversal agents and specific reversal agents.

\section{A. Nonspecific Reversal Agents}

Multiple nonspecific prothrombotic preparations have been studied in the reversal of DOAC-based anticoagulation. These agents were used in emergent settings, before the advent of specific reversal agents.

\section{Prothrombin Complex Concentrates/Activated} Prothrombic Complex Concentrates. Prothrombic complex concentrates (PCCs) are derived from plasma and contain 3 to 4 clotting factors (factor II, IX, X, and VII), protein $\mathrm{C}$ and protein $\mathrm{S}$, and heparin. Activated PCC (aPCC) consists of a large proportion of activated factor VII in addition to nonactivated factors II, IX, and X. Although initial data from animal studies demonstrated a reduction in bleeding events in all DOAC classes, ${ }^{55,56}$ data in human subjects remain very limited. PCC or aPCC at a dose of $50 \mathrm{U} / \mathrm{kg}$ is recommended for the reversal of DOAC-based anticoagulation in emergent clinical settings (if a specific reversal agent is unavailable). ${ }^{57}$

II. Fresh Frozen Plasma and Vitamin K. There is no role for the use of fresh frozen plasma or vitamin $\mathrm{K}$ as reversal agents for DOAC agents. ${ }^{57}$ 
III. Recombinant Activated Factor VII. A limited number of small studies suggested activated factor VII as a reversal agent in severe DOAC-related bleeding. Unfortunately, no there are no clinical trials that support this notion. ${ }^{58}$

Overall, there is very limited evidence to support the efficacy and safety of these nonspecific reversal agents in the setting of DOAC-based anticoagulation. These limited data involve normalizing coagulation parameters in otherwise healthy subjects and did not analyze important clinical end points. Furthermore, evidence from the use of these agents in the setting of VKA and hemophilia-related bleeding events suggests that there is an associated increased risk for thrombotic complications under these circumstances. ${ }^{59,60}$

\section{B. Specific Reversal Agents}

The advent of specific reversal agents for DOAC therapy has added considerable strength to the argument in favor of the use of DOAC agents. These agents address one of the most anxiety-provoking shortcomings of this new class of anticoagulants: the need for fast and reliable reversal in emergent settings. Idarucizumab is the only commercially available agent. However, another reversal agent, andexanet alfa, has been approved by the FDA and is expected to be commercially available in the coming months. Ciraparantag is another specific DOAC-reversal agent that is in the early stages of development but has shown promising results.

I. Idarucizumab. Idarucizumab is a humanized, monoclonal antibody fragment that was developed as a specific reversal agent for the direct thrombin inhibitor, dabigatran. In animal studies, idarucizumab reduced blood loss in a dose-dependent manner. ${ }^{61}$ As an antibody fragment, it binds to dabigatran (free and thrombin bound). This bind- ing reverses the anticoagulant effect of dabigatran in human volunteers of varied clinical profiles, including the elderly and those with renal impairment. ${ }^{62,63}$ Furthermore, idarucizumab administered in the absence of dabigatran had no effect on coagulation parameters or endogenous thrombin generation. ${ }^{62}$ Preliminary findings of the RE-VERSE AD trial demonstrated a rapid normalization of coagulation parameters in patients anticoagulated with dabigatran. ${ }^{64}$ Given the significant need for such an agent, the FDA granted accelerated approval to idarucizumab to reverse anticoagulation with dabigatran in emergent situations in October 2015. Currently, idarucizumab remains the only commercially available specific reversal agent for a DOAC agent.

II. Andexanet Alfa. Andexanet alfa is a modified human recombinant factor $\mathrm{Xa}$ decoy protein designed to reverse factor Xa inhibitors. It is expected to be effective for both direct (apixaban, edoxaban, and rivaroxaban) and indirect (heparin and fondaparinux) factor Xa inhibitors. Andexanet effectively binds factor $\mathrm{Xa}$ inhibitors, sequestering them within the vascular space. ${ }^{65}$ This leads to the restoration of endogenous factor Xa activity, demonstrated by decreased antifactor Xa activity and increased thrombin generation. ${ }^{66}$ Andexanet showed rapid, dose-dependent reversal of anticoagulation in healthy volunteers (receiving apixaban, rivaroxaban, and enoxaparin) in phase 2 studies. ${ }^{67-69}$ In 2 subsequent randomized trials, ANNEXA-A and ANNEXA-R, andexanet rapidly reduced antifactor $\mathrm{Xa}$ activity (within 2 to 5 minutes) in volunteers receiving apixaban (ANNEXA-A) or rivaroxaban (ANNEXA-R). Thrombin generation was also rapidly and fully restored in nearly all participants. No thrombotic or other serious adverse events were reported..$^{65}$

Table 3. Direct Oral Anticoagulant Characteristics: Interruption and Reversal ${ }^{54}$

\begin{tabular}{lllll}
\hline Characteristics & \multicolumn{1}{c}{ Dabigatran } & Rivaroxaban & Apixaban & Edoxaban \\
\hline Mechanism & Ila inhibitor & Xa inhibitor & Xa inhibitor & Xa inhibitor \\
Specific reversal agents (first line) & Praxbind (idarucizumab) & Andexanet & Andexanet & Andexanet \\
& & Alfa* & Alfa* & Alfa* \\
& & Ciraparantag* & Ciraparantag* & Ciraparantag* \\
Second line reversal agents & aPCC & aPCC & aPCC & aPCC \\
& 4F- PCC & & & \\
\hline
\end{tabular}

aPCC, activated prothrombin complex concentrate; $4 \mathrm{~F}-\mathrm{PCC}=4$ factor prothrombin complex concentrate.

*Not commercially available in the USA. 
ANNEXA-4 is a prospective, open-label study that is currently recruiting patients to assess the efficacy and safety of andexanet in patients on a factor $\mathrm{Xa}$ inhibitor with acute major bleeding (http://www.clinicaltrials.gov, NCT02329327). All patients included in the study presented with an acute bleeding event following the administration of an oral factor $\mathrm{Xa}$ inhibitor. As an intervention, they received a 2-hour infusion of andexanet alfa. An interim analysis of the ANNEXA-4 study was presented at the American College of Cardiology Scientific Sessions in Orlando, FL, on March 2018, as a late-breaking trial. Interim data on safety outcomes of 227 patients and efficacy outcomes of 132 patients were presented. Excellent or good clinical hemostasis was achieved in $83 \%$ of the patients. At 30 days, $12 \%$ of the patients were deceased and $11 \%$ had experienced a thrombotic episode. The authors contend that these safety outcomes are consistent with expectations in this high-risk study group. Details of these safety and efficacy outcomes are expected to be included in the final analysis at the time of publication.

Andexanet remains under consideration by the European Medicines Agency. Most recently, on May 4, 2018, the FDA granted approval for the use of andexanet alfa. The commercial launch of the drug is expected later this year.

III. Ciraparantag. Ciraparantag is a small, synthetic water-soluble molecule that binds to factor $\mathrm{Xa}$ inhibitors through noncovalent hydrogen bonding and charge-charge interactions, thereby preventing the anticoagulants from binding to their endogenous targets. It is being developed as a reversal agent for oral direct factor $\mathrm{Xa}$ and heparin (unfractionated and low molecular weight preparations), argatroban, and fondaparinux. Animal studies demonstrated a potent reversal of DOAC-based anticoagulation with a reduction in hemorrhage and restoration of coagulation parameters. ${ }^{70,71}$ In a phase 1 trial with healthy volunteers receiving edoxaban, ciraparantag decreased whole-blood clotting times to within $10 \%$ of baseline values within 10 minutes, and this reversal was sustained for 24 hours. No thrombotic events or other adverse events were reported. ${ }^{72}$ Further study of this promising reversal agent is ongoing.

Overall, the management of DOAC-based anticoagulation in the preprocedural and acute care settings is a challenge that requires a nuanced ap- proach. A summary of the considerations that guide decision making in these settings is a useful tool (Table 3). The safety and efficacy of specific reversal agents need further analysis in the clinical setting. When multiple reversal agents become available, an evidence-based guideline document with specific algorithms regarding their use in acute settings will be required.

\section{Conclusion}

1. The increasing prevalence of DOAC-based anticoagulation necessitates discussion and planning for complications and emergencies.

2. In the past, the absence of specific reversal agents made DOACs less attractive to patients and physicians. Furthermore, nonspecific reversal agents have been less than satisfactory in the acute setting.

3. Currently, idarucizumab is the only commercially available reversal agent and is effective against dabigatran.

4. Multiple reversal agents are under development. Their progress is under active review by the FDA.

5. Andexanet alfa is a reversal agent (under development) that is targeted toward factor $\mathrm{Xa}$ inhibitors (oral and parenteral). Ciraparantag is another agent under development that has the potential to reverse all DOACs and most parenteral anticoagulants.

6. The management of DOAC-based anticoagulation in the preprocedural and acute care settings is a challenge that requires a nuanced approach and individualization based on patient characteristics and clinical settings.

To see this article online, please go to: http://jabfm.org/content/ 31/5/817.full.

\section{References}

1. Desai NR, Krumme AA, Schneeweiss S, et al. Patterns of initiation of oral anticoagulants in patients with atrial fibrillation-quality and cost implications. Am J Med 2014;127:1075-82.e1.

2. Lane DA, Lip GYH. Use of the CHA2DS2-VASc and HAS-BLED scores to aid decision making for thromboprophylaxis in nonvalvular atrial fibrillation. Circulation 2012;126:860-5.

3. You JJ, Singer DE, Howard PA, et al. Antithrombotic therapy for atrial fibrillation: antithrombotic therapy and prevention of thrombosis, 9th ed: American College of Chest Physicians evidence-based 
clinical practice guidelines. Chest 2012;141:e531Se575S.

4. Camm AJ, Kirchhof P, Lip GYH, et al. Guidelines for the management of atrial fibrillation: the Task Force for the Management of Atrial Fibrillation of the European Society of Cardiology (ESC). Eur Heart J 2010;31:2369-429.

5. January CT, Wann LS, Alpert JS, et al. 2014 AHA/ ACC/HRS guideline for the management of patients with atrial fibrillation: executive summary. J Am Coll Cardiol 2014;64:2246-80.

6. Connolly SJ, Ezekowitz MD, Yusuf S, et al. Dabigatran versus warfarin in patients with atrial fibrillation. N Engl J Med 2009;361:1139-51.

7. Patel MR, Mahaffey KW, Garg J, et al. Rivaroxaban versus warfarin in nonvalvular atrial fibrillation. N Engl J Med 2011;365:883-91.

8. Connolly SJ, Eikelboom J, Joyner C, et al. Apixaban in patients with atrial fibrillation. N Engl J Med 2011;364:806-17.

9. Granger CB, Alexander JH, McMurray JJV, et al. Apixaban versus warfarin in patients with atrial fibrillation. N Engl J Med 2011;365:981-92.

10. $\mathrm{Ng} \mathrm{KH,} \mathrm{Shestakovska} \mathrm{O,} \mathrm{Connolly} \mathrm{SJ,} \mathrm{et} \mathrm{al.} \mathrm{Efficacy}$ and safety of apixaban compared with aspirin in the elderly: a subgroup analysis from the AVERROES trial. Age Ageing 2016;45:77-83.

11. Giugliano RP, Ruff CT, Braunwald E, et al. Edoxaban versus warfarin in patients with atrial fibrillation. N Engl J Med 2013;369:2093-104.

12. Diener HC, Aisenberg J, Ansell J, et al. Choosing a particular oral anticoagulant and dose for stroke prevention in individual patients with non-valvular atrial fibrillation: part 1. Eur Heart J 2017;38:852-9.

13. Diener HC, Aisenberg J, Ansell J, et al. Choosing a particular oral anticoagulant and dose for stroke prevention in individual patients with non-valvular atrial fibrillation: part 2. Eur Heart J 2017;38:860-8.

14. Urooj F, Kulkarni A, Stapleton D, Kaluski E. New oral anticoagulants in nonvalvular atrial fibrillation. Clin Cardiol 2016;39:739-46.

15. EINSTEIN Investigators, Bauersachs R, Berkowitz $\mathrm{SD}$, et al. Oral rivaroxaban for symptomatic venous thromboembolism. N Engl J Med 2010;363:2499510.

16. EINSTEIN-PE Investigators, Büller HR, Prins $\mathrm{MH}$, et al. Oral rivaroxaban for the treatment of symptomatic pulmonary embolism. N Engl J Med 2012;366:1287-97.

17. Agnelli G, Buller HR, Cohen A, et al. Oral apixaban for the treatment of acute venous thromboembolism. N Engl J Med 2013;369:799-808.

18. Hokusai-VTE Investigators, Büller HR, Décousus $\mathrm{H}$, et al. Edoxaban versus warfarin for the treatment of symptomatic venous thromboembolism. N Engl J Med 2013;369:1406-15.

19. Schulman S, Kearon C, Kakkar AK, et al. Dabigatran versus warfarin in the treatment of acute ve- nous thromboembolism. N Engl J Med 2009;361: 2342-52.

20. Gould MK, Garcia DA, Wren SM, et al. Prevention of VTE in nonorthopedic surgical patients: antithrombotic therapy and prevention of thrombosis, 9th ed: American College of Chest Physicians evidence-based clinical practice guidelines. Chest 2012; 141:e227S-e277S.

21. Falck-Ytter Y, Francis CW, Johanson NA, et al. Prevention of VTE in orthopedic surgery patients: antithrombotic therapy and prevention of thrombosis, 9th ed: American College of Chest Physicians evidence-based clinical practice guidelines. Chest 2012;141:e278S-e325S.

22. Kahn SR, Lim W, Dunn AS, et al. Prevention of VTE in nonsurgical patients: antithrombotic therapy and prevention of thrombosis, 9th ed: American College of Chest Physicians evidence-based clinical practice guidelines. Chest 2012;141:e195S-e226S.

23. Gibson CM, Halaby R, Korjian S, et al. The safety and efficacy of full- versus reduced-dose betrixaban in the Acute Medically Ill VTE (venous thromboembolism) Prevention with Extended-Duration Betrixaban (APEX) trial. Am Heart J 2017;185:93-100.

24. Senoo K, Lane D, Lip GYH. Stroke and bleeding risk in atrial fibrillation. Korean Circ J 2014;44: 281-90.

25. Apostolakis S, Lane DA, Guo Y, Buller H, Lip GYH. Performance of the HEMORR 2HAGES, ATRIA, and HAS-BLED bleeding risk-prediction scores in patients with atrial fibrillation undergoing anticoagulation: the AMADEUS (Evaluating the use of SR34006 compared to warfarin or acenocoumarol in patients with atrial. J Am Coll Cardiol 2012;60: 861-7.

26. Roldán V, Marín F, Fernández H, et al. Predictive value of the HAS-BLED and ATRIA bleeding scores for the risk of serious bleeding in a "real-world" population with atrial fibrillation receiving anticoagulant therapy. Chest 2013;143:179-84.

27. Kearon C, Akl EA, Ornelas J, et al. Antithrombotic therapy for VTE disease. Chest 2016;149:315-52.

28. Van Es N, Coppens M, Schulman S, Middeldorp S, Büller HR. Direct oral anticoagulants compared with vitamin $\mathrm{K}$ antagonists for acute venous thromboembolism: evidence from phase 3 trials. Blood 2014;124:1968-75.

29. Chai-Adisaksopha C, Crowther M, Isayama T, Lim $W$. The impact of bleeding complications in patients receiving target-specific oral anticoagulants: a systematic review and meta-analysis. Blood 2014;124: 2450-8.

30. Abraham NS, Singh S, Alexander GC, et al. Comparative risk of gastrointestinal bleeding with dabigatran, rivaroxaban, and warfarin: population based cohort study. BMJ 2015;350:h1857.

31. Holster IL, Valkhoff VE, Kuipers EJ, Tjwa ETTL. New oral anticoagulants increase risk for gastroin- 
testinal bleeding: a systematic review and meta-analysis. Gastroenterology 2013;145:105-12.e15.

32. Castellucci LA, Cameron C, Le Gal G, et al. Clinical and safety outcomes associated with treatment of acute venous thromboembolism: a systematic review and meta-analysis. JAMA 2014;312:1122-35.

33. January CT, Wann LS, Alpert JS, et al. 2014 AHA/ ACC/HRS guideline for the management of patients with atrial fibrillation: a report of the American College of cardiology/American Heart Association task force on practice guidelines and the heart rhythm society. Circulation 2014;130:e199-e267.

34. Wang X, Tirucherai G, Marbury TC, et al. Pharmacokinetics, pharmacodynamics, and safety of apixaban in subjects with end-stage renal disease on hemodialysis. J Clin Pharmacol 2016;56:628-36.

35. Douketis JD, Berger PB, Dunn AS, et al. The perioperative management of antithrombotic therapy: American College of Chest Physicians evidencebased clinical practice guidelines (8th ed.). Chest 2008;133:299S-339S.

36. Kovacs RJ, Flaker GC, Saxonhouse SJ, et al. Practical management of anticoagulation in patients with atrial fibrillation. J Am Coll Cardiol 2015;65: $1340-60$.

37. Patel IJ, Davidson JC, Nikolic B, et al. Consensus guidelines for periprocedural management of coagulation status and hemostasis risk in percutaneous image-guided interventions. J Vasc Interv Radiol 2012;23:727-36.

38. Burnett AE, Mahan CE, Vazquez SR, Oertel LB, Garcia DA, Ansell J. Guidance for the practical management of the direct oral anticoagulants (DOACs) in VTE treatment. J Thromb Thrombolysis 2016; 41:206-32.

39. Gage BF, Yan Y, Milligan PE, et al. Clinical classification schemes for predicting hemorrhage: results from the National Registry of Atrial Fibrillation (NRAF). Am Heart J 2006;151:713-9.

40. Grove EL, Hossain R, Storey RF. Platelet function testing and prediction of procedural bleeding risk. Thromb Haemost 2013;109:817-24.

41. Singer DE, Chang Y, Borowsky LH, et al. A new risk scheme to predict ischemic stroke and other thromboembolism in atrial fibrillation: the ATRIA study stroke risk score. J Am Heart Assoc 2013;2: $\mathrm{e} 000250$.

42. Douketis JD, Spyropoulos AC, Spencer FA, et al. Perioperative management of antithrombotic therapy. Antithrombotic therapy and prevention of thrombosis, 9th ed: American College of Chest Physicians evidence-based clinical practice guidelines. Chest 2012;141:e326S-e350S.

43. Doherty JU, Gluckman TJ, Hucker WJ, et al. 2017 ACC expert consensus decision pathway for periprocedural management of anticoagulation in patients with nonvalvular atrial fibrillation. J Am Coll Cardiol 2017;69:871-98.
44. Stangier J, Rathgen K, Sthle H, Mazur D. Influence of renal impairment on the pharmacokinetics and pharmacodynamics of oral dabigatran etexilate: an open-label, parallel-group, single-centre study. Clin Pharmacokinet 2010;49:259-68.

45. Schulman S, Carrier M, Lee AYY, et al. Perioperative management of dabigatran: a prospective cohort study. Circulation 2015;132:167-73.

46. Van Ryn J, Stangier J, Haertter S, et al. Dabigatran etexilate - a novel, reversible, oral direct thrombin inhibitor: interpretation of coagulation assays and reversal of anticoagulant activity. Thromb Haemost 2010;103:1116-27.

47. Spyropoulos AC, Douketis JD. How I treat anticoagulated patients undergoing an elective procedure or surgery. Blood 2012;120:2954-62.

48. Narouze S, Benzon HT, Provenzano D, et al. Interventional spine and pain procedures in patients on antiplatelet and anticoagulant medications (second edition): guidelines from the American Society of Regional Anesthesia and Pain Medicine, the European Society of Regional Anaesthesia and Pain Therapy, the American Academy of Pain Medicine, the International Neuromodulation Society, the North American Neuromodulation Society, and the World Institute of Pain. Reg Anesth Pain Med 2018; 43:225-262.

49. Douketis JD, Spyropoulos AC, Kaatz S, et al. Perioperative bridging anticoagulation in patients with atrial fibrillation. N Engl J Med 2015;373:823-33.

50. Zalpour A, Oo TH. Update on edoxaban for the prevention and treatment of thromboembolism: clinical applications based on current evidence. Adv Hematol 2015;2015.

51. Lane DA, Aguinaga L, Blomström-Lundqvist C, et al. Cardiac tachyarrhythmias and patient values and preferences for their management: the European Heart Rhythm Association (EHRA) consensus document endorsed by the Heart Rhythm Society (HRS), Asia Pacific Heart Rhythm Society (APHRS), and Sociedad Latinoame. EP Eur 2015; 17:1747-69.

52. Aronis KN, Hylek EM. Who, when, and how to reverse non-vitamin $\mathrm{K}$ oral anticoagulants. J Thromb Thrombolysis 2016;41:253-72.

53. Wanek MR, Horn ET, Elapavaluru S, Baroody SC, Sokos G. Safe use of hemodialysis for dabigatran removal before cardiac surgery. Ann Pharmacother 2012;46:e21.

54. Tomaselli G, Mahaffey K, Cuker A, et al. 2017 ACC expert consensus decision pathway on management of bleeding in patients on oral anticoagulants: a report of the American College of Cardiology task force on expert consensus decision pathways. J Am Coll Cardiol 2017;70:3042-3067.

55. Weitz JI, Pollack CV. Practical management of bleeding in patients receiving non-vitamin $\mathrm{K}$ antag- 
onist oral anticoagulants. Thromb Haemost 2015; 114:1113-26.

56. Godier A, Miclot A, Le Bonniec B, et al. Evaluation of prothrombin complex concentrate and recombinant activated factor VII to reverse rivaroxaban in a rabbit model. Anesthesiology 2012;116:94-102.

57. Heidbuchel H, Verhamme P, Alings M, et al. Updated European Heart Rhythm Association practical guide on the use of non-vitamin $\mathrm{K}$ antagonist anticoagulants in patients with non-valvular atrial fibrillation. Europace 2015;17:1467-507.

58. Ruff CT, Giugliano RP, Antman EM. Management of bleeding with non-vitamin $\mathrm{K}$ antagonist oral anticoagulants in the era of specific reversal agents. Circulation 2016;134:248-61.

59. Dentali F, Marchesi C, Pierfranceschi MG, et al. Safety of prothrombin complex concentrates for rapid anticoagulation reversal of vitamin $\mathrm{K}$ antagonists. Thromb Haemost 2011;106:429-38.

60. Ehrlich HJ, Henzl MJ, Gomperts ED. Safety of factor VIII inhibitor bypass activity (FEIBA): 10year compilation of thrombotic adverse events. Haemophilia 2002;8:83-90.

61. van Ryn J, Litzenburger T, Gan G, Coble K, Schurer $\mathrm{J}$. In vitro characterization, pharmacokinetics and reversal of supratherapeutic doses of dabigatran-induced bleeding in rats by a specific antibody fragment antidote to dabigatran. Blood 2012;120:3418.

62. Glund S, Moschetti V, Norris S, et al. A randomised study in healthy volunteers to investigate the safety, tolerability and pharmacokinetics of idarucizumab, a specific antidote to dabigatran. Thromb Haemost 2015;113:943-51.

63. Glund S, Stangier J, Schmohl M, et al. Idarucizumab, a specific antidote for dabigatran: immediate, complete and sustained reversal of dabigatran induced anticoagulation in elderly and renally impaired subjects. Blood 2014;124:344-344.

64. Pollack CV, Reilly PA, van Ryn J, et al. Idarucizumab for dabigatran reversal - full cohort analysis. N Engl J Med 2017;377:431-41.

65. Siegal DM, Curnutte JT, Connolly SJ, et al. Andexanet alfa for the reversal of factor Xa inhibitor activity. N Engl J Med 2015;373:2413-24.

66. Lu G, Deguzman FR, Hollenbach SJ, et al. A specific antidote for reversal of anticoagulation by direct and indirect inhibitors of coagulation factor Xa. Nat Med 2013;19:446-51.

67. Crowther M, Lu G, Conley P, et al. Reversal of factor XA inhibitors-induced anticoagulation in healthy subjects by andexanet ALFA. Crit Care Med 2014;42:A1469.

68. Vandana M, Michael K, Genmin L, et al. A phase 2 randomized, double-blind, placebo-controlled trial demonstrating reversal of rivaroxaban-induced anticoagulation in healthy subjects by andexanet alfa (PRT064445), an antidote for fxa inhibitors. Blood 2013;122:3636.

69. Crowther M, Kitt M, Lorenz T, et al. A phase 2 randomized, double-blind, placebo-controlled trial of PRT064445, a novel, universal antidote for direct and indirect factor Xa inhibitors: AS 20.1. J Thromb Haemost 2013;11:30.

70. Laulicht B, Bakhru S, Jiang X, et al. Antidote for new oral anticoagulants: mechanism of action and binding specificity of PER977. Thromb Haemost 2013; 11:75.

71. Laulicht B, Bakhru S, Lee C, Baker C. Small molecule antidote for anticoagulants. Circulation 2012; 126:10021.

72. Ansell JE, Bakhru SH, Laulicht BE, et al. Use of PER977 to reverse the anticoagulant effect of edoxaban. N Engl J Med 2014;371:2141-2.

73. Kubitza D, Becka M, Mueck W, et al. Effects of renal impairment on the pharmacokinetics, pharmacodynamics and safety of rivaroxaban, an oral, direct Factor Xa inhibitor. Br J Clin Pharmacol 2010;70: 703-12.

74. Parasrampuria DA, Marbury T, Matsushima N, et al. Pharmacokinetics, safety, and tolerability of edoxaban in end-stage renal disease subjects undergoing haemodialysis. Thromb Haemost 2015;113: 719-27.

75. Dias C, Moore KT, Murphy J, et al. Pharmacokinetics, pharmacodynamics, and safety of single-dose rivaroxaban in chronic hemodialysis. Am J Nephrol 2016;43:229-36.

76. Chang M, Yu Z, Shenker A, et al. Effect of renal impairment on the pharmacokinetics, pharmacodynamics, and safety of apixaban. J Clin Pharmacol 2016;56:637-45. 